\title{
The Effect of Convective Overstability on Planet Disk Interactions
}

\author{
Hubert Klahr ${ }^{1}$ and Aiara Lobo Gomes ${ }^{2}$ \\ Max-Planck-Institut für Astronomie, \\ Königstuhl 17, 69117 Heidelberg, Germany \\ ${ }^{1}$ email: klahr@mpia.de \\ ${ }^{2}$ email: gomes@mpia.de
}

\begin{abstract}
We run global two dimensional hydrodynamical simulations, using the PLUTO code and the planet-disk model of Uribe et al. 2011, to investigate the effect of the convective overstability $(\mathrm{CO})$ on planet-disk interactions. First, we study the long-term evolution of planetinduced vortices. We found that the $\mathrm{CO}$ leads to smoother planetary gap edges, thus weaker planet-induced vortices. The main result was the observation of two generation of vortices, which can pose an explanation for the location of the vortex in the Oph IRS48 system. The lifetime of the primary vortices, as well as the birth time of the secondary vortices are shown to be highly dependent on the thermal relaxation timescale. Second, we study the long-term evolution of the migration of low mass planets and assess whether the $\mathrm{CO}$ can prevent the saturation of the horseshoe drag. We found that the disk parameters that favour slow inward or outward migration oppose the amplification of vortices, meaning that the $\mathrm{CO}$ does not seem to be a good mechanism to prevent the saturation of the horseshoe drag. On the other hand, we observed a planetary trap, caused by vortices formed in the horseshoe region. This trap may be an alternative mechanism to prevent the fast type I migration rates.
\end{abstract}

Keywords. Accretion disks, hydrodynamics, instabilities - Methods: numerical - Planetary systems: protoplanetary disks

\section{Introduction}

The magnetorotational instability (MRI, Balbus \& Hawley 1991) is the most invoked mechanism to explain turbulence in protoplanetary disks (PPDs). Nevertheless, hydrodynamical (HD) instabilities are also shown to be able to produce turbulence, especially in regions of the disk with too low ionisation levels to couple efficiently to the magnetic fields, so called Dead Zones. For instance, vortices generated by the Rossby wave instability (RWI, Lovelace et al. 1999) are good candidates to create vortices at the dead zone edge (Lyra \& Mac Low 2012). The baroclinic instability (BI, Klahr \& Bodenheimer 2003) leads also to vortex formation and thermal relaxation or diffusion are important ingredients for vortex amplification (Petersen et al. 2007a,b). The non-linear phase of baroclinic vortex amplification is coined as subcritical baroclinic instability (SBI, Lesur \& Papaloizou 2010), whereas the linear phase is the convective overstability (CO, Klahr \& Hubbard 2014). Hence we claim that BI, SBI and CO are all the same phenomenon, i.e. radial convection in a rapidly rotating medium with the proper thermal relaxation, and in call the phenomenon CO. CO does occur for disks with superadiabatic radial stratification, that is pressure gradient $\left(\beta_{P}=\frac{d \log P}{d \log R}\right)$ and entropy gradients $\left(\beta_{S}=\frac{d \log S}{d \log R}\right)$ pointing in the same direction. In the two-dimensional vertically integrated case they can be derived from the radial temperature $\left(\beta_{T}=\frac{d \log T}{d \log R}\right)$ and surface density profile $\left(\beta_{T}=\frac{d \log T}{d \log R}\right)$ to $\beta_{P}=\beta_{T}+\beta_{\Sigma}$ and using the adiabatic index of $\gamma=1.345$ to $\beta_{S}=\beta_{T}-(\gamma-1) \beta_{\Sigma}$. The maximum growth-rates for optimal thermal relaxation are then proportional to the 
local pressure scale height $h=H / R$ and proportional to the radial buoyancy frequency $N^{2}=-\beta_{S} \beta_{P} h^{2}$. Thus as long as surfacedensity and temperature fall with radius and the surfacedensity slope is shallower than $\beta_{\Sigma}=\frac{\beta_{T}}{(\gamma-1)}$ then the disk is radially buoyant and provided that the thermal relaxation occurs on timescales on the order of the local orbital period the instabilities will develop and create vortices (see Raettig et al. 2013 for a detailed exploration). For a temperature profile of $\beta_{T}=-0.5$ the radial surface density profile has to be shallower than $\beta_{\Sigma}>-1.44$, values that can easily be found in disks around young stars (Andrews et al. 2010).

Whereas the detailed properties of global $3 \mathrm{D}$ turbulence driven by the $\mathrm{CO}$ is still poorly understood for the lack of vertically stratified simulations, it is obvious the flow is dominated by large scale vortices and large scale density waves in between, with the latter providing Reynolds stresses on the order of $\alpha \approx 10^{-3}$ (Lyra \& Klahr 2011; Raettig et al. 2013).

Here, we discuss the effect of the $\mathrm{CO} / \mathrm{SBI}$ on planet-disk interactions for high and low mass planets, i.e. planets that can open a gap or can not. The formation and evolution of vortices induced by high mass planets have recently been thoroughly investigated (e.g., Zhu \& Stone 2014; Fu et al. 2014; Les \& Lin 2015). Vortices are important structures in several contexts. They are good candidates to trap dust particles, preventing them to quickly drift towards the central star and allowing them to grow to bigger sizes (Barge \& Sommeria 1995; Klahr \& Bodenheimer 2006). Therefore they are a good channel to overcome the radial drift barrier (Whipple 1972) for planet formation. They may also explain angular momentum transport through the disk dead zone, which is essential to allow for inward accretion (e.g., Meheut et al. 2012). The exchange of angular momentum between waves in the inner and outer side of the vortex position leads to a negative net flux of angular momentum and therefore a positive flux of mass. Finally, vortices may also trap planet cores, slowing down the core migration towards the central star, easing the timescale problem of the rapid inward drift of small objects (e.g., Koller et al. 2003; Ou et al. 2007; Li et al. 2009; Yu et al. 2010; Regály et al. 2013; Ataiee et al. 2014).

Type I migration is another aspect of planet-disk interactions worth investigating in this context. Low mass planets typically experience type I migration (Ward 1997; Tanaka et al. 2002), which is governed by the Lindblad torque (Ward 1986, 1988) and the horseshoe drag (e.g., Paardekooper \& Papaloizou 2009; Casoli \& Masset 2009). The former being the gravitational torque due to spiral density waves launched by the planet. The latter being the torque exerted by fluid elements executing a U-turn around the planet. A problem related to type I migration is the timescales predicted by isothermal models, which are much shorter than the typical disk lifetimes (Ward 1986). It would not be possible to explain the observed mass-distance distribution of exoplanets with such fast migration rates (Alibert et al. 2004; Ida \& Lin 2008; Mordasini et al. 2009). Recent works have shown that non-isothermal effects can increase the horseshoe drag, which leads to slower migration rates or even the reversal of the migration direction (e.g., Morohoshi \& Tanaka 2003; Paardekooper \& Mellema 2006; Baruteau \& Masset 2008). Nevertheless, there are still questions related to this subject. How can the saturation of the horseshoe drag be prevented, such that slow type I migration is sustained? This is an important question to address. In the next sections we summarise the main results and conclusions for simulations considering high and low mass planets.

\section{High Mass Planets}

High mass planets are here defined as planets massive enough to open a gap in the disk and thus will be subject of TypeII migration. The critical mass lies around the mass 


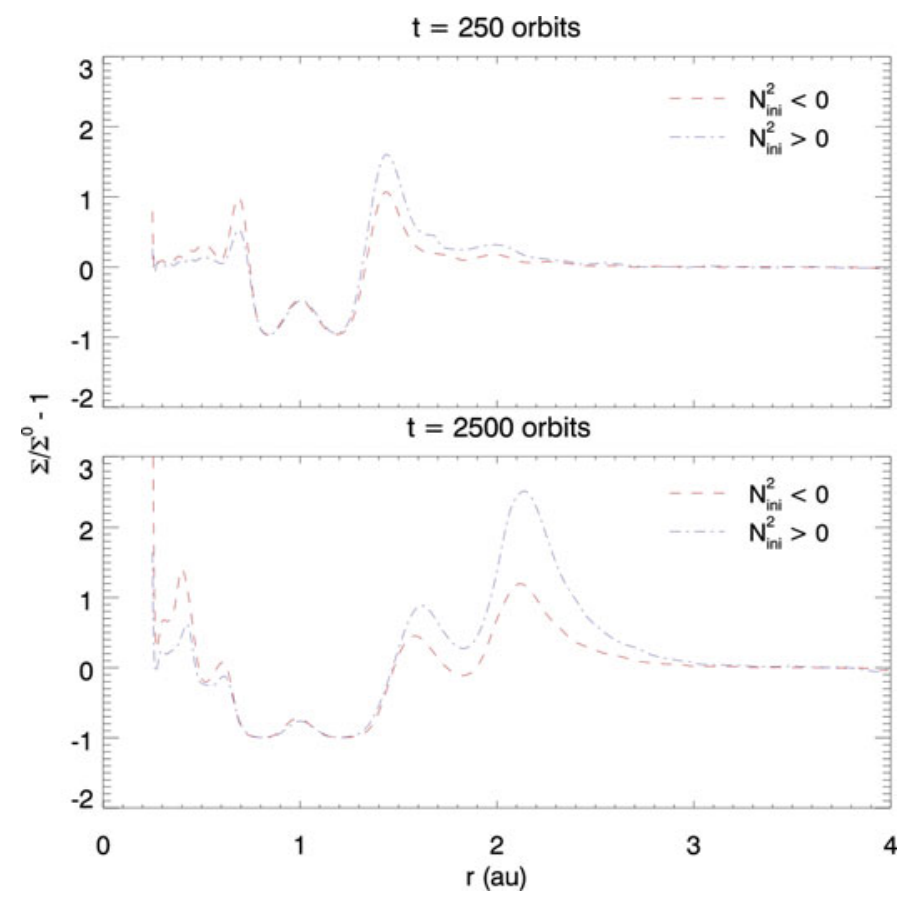

Figure 1. Surface density profiles averaged in azimuth for a disk with a thermal relaxation time of $\Omega \tau=0.1$ in the presence a Jupiter mass planet. The red dashed line shows the results for a initially convective overstable disk profile, whereas the blue dotted-dashed line for a initially convective stable disk profile. In the upper plot one finds one vortex (after 250 orbits) and after 2500 orbits in lower plot there are two vortices, with the density perturbation stronger for the stable stratified disk.

of Saturn (in detail depending on the disk temperature), i.e. $100 M_{\oplus}$. For the present study we use a mass of $300 M_{\oplus}$ i.e. a Jupiter mass planet.

Dust asymmetries have recently been observed in several transition disks, e.g., in the Oph IRS 48 system (van der Marel et al. 2013). These features may be explained by the presence of large-scale vortices born at the outer edge of a planetary gap. Motivated by these observations, several recent studies have focused on the formation and evolution of planet-induced vortices (e.g., Zhu \& Stone 2014; Fu et al. 2014; Les \& Lin 2015). Questions regarding the lifetimes of these features and whether they are able to trap dust particles are fundamental to check if they can explain these recent observations. We performed two-dimensional (2D) global inviscid HD simulations to investigate planetinduced vortices (Lobo Gomes et al. 2015). A Newtonian cooling approach (thermal relaxation) was used to evolve the disk temperature structure. The main aim was to assess the importance of the $\mathrm{CO}$ for the development of planet-induced vortices. We found that the CO leads to smoother planetary gap edges, therefore weaker vortices are generated, see Figure 1. An interesting outcome of this study was the observation of a second generation of vortices. The first generation of vortices is formed in the outer wall of the planetary gap due to the triggering of the RWI.

The RWI is here responsible for the initial creation of a vortex whereas the CO will govern its evolution, i.e. amplify or damp the vortex depending on the local radial stratification and the thermal relaxation time.

The merged primary vortex induces accretion, which depletes the mass on its orbit. This process creates a surface density enhancement beyond the primary vortex position. 


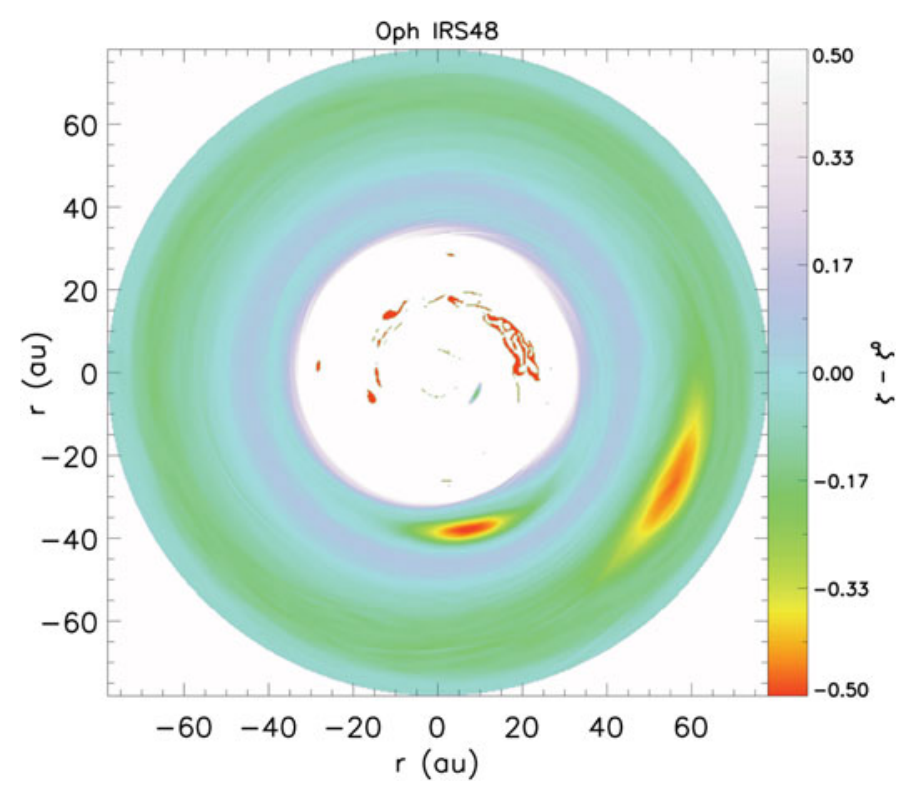

Figure 2. Potential vorticity with the Keplerian profile subtracted after 700 planetary orbits.

The color bar was truncated from -0.5 to 0.5 in order to obtain a higher contrast.

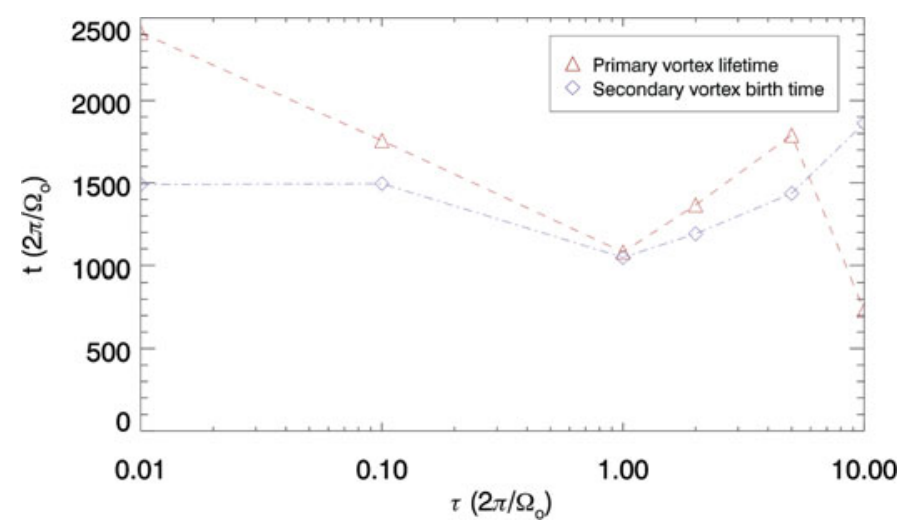

Figure 3. The lifetime of the primary vortex (red dashed line) and the birth time of the secondary vortex (blue dotted-dashed line) as a function of the thermal relaxation timescale.

The second generation of vortices arises in this surface density enhancement, indicating that the bump in this region is sufficient to trigger the RWI once more. We modelled the Oph IRS48 system with the objective of checking whether the second generation of vortices would also be formed. The hypothesis that a planet-induced vortex is the reason for the asymmetric feature in this system has a major problem: the location of the asymmetry cannot be explained by a vortex at the planetary gap edge, since the vortex location is very far from the possible planet location. We observed a second generation of vortices in the model of this system, therefore posing a promising explanation for the location of the vortex, see Figure 2.

We also studied the dependence of the vortices lifetimes and birth times as a function of the thermal relaxation timescale. The primary vortices' lifetimes, as well as the secondary vortices' birth times, were found to be dependent on the disk thermal relaxation 


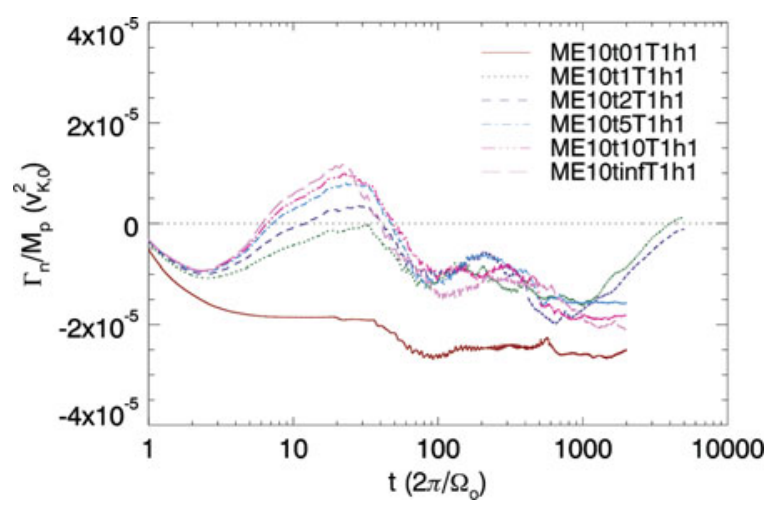

Figure 4. Cumulative averaged torques as a function of time. The different lines show the total torques for a $10 M_{\oplus}$ planet, $\beta_{T}=1.0, h=0.1$, and the thermal relaxation timescales from bottom to top (solid brown to dashed pink): $\Omega \tau=[0.1,1.0,2,5,10, \infty]$. See Table 1 in Lobo Gomes et al. (submitted) for the details of the runs.

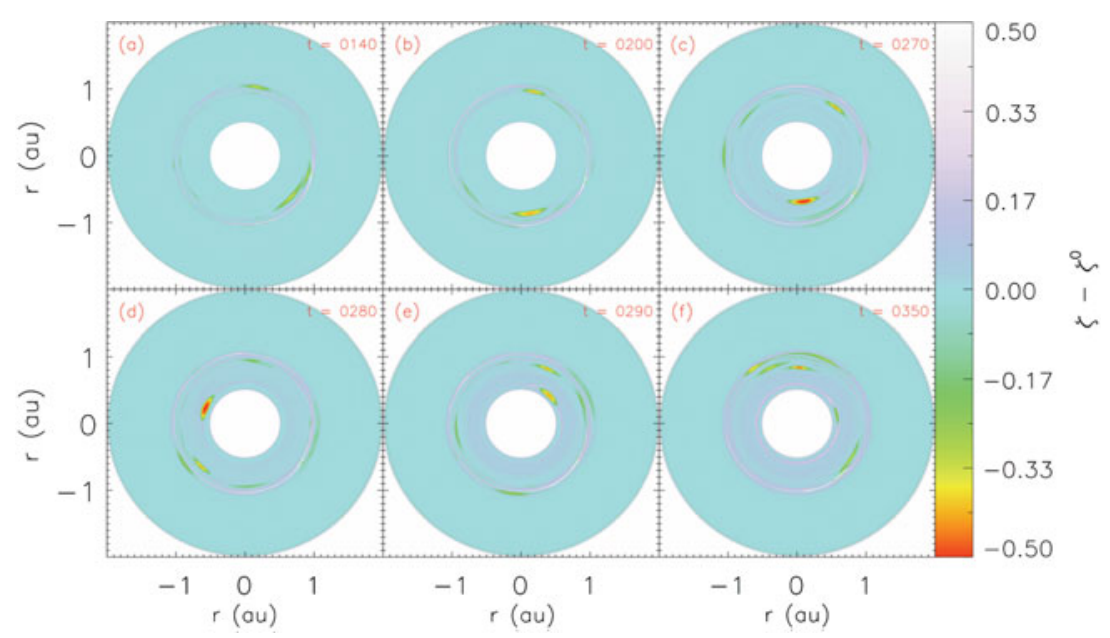

Figure 5. Temporal evolution of the potential vorticity with Keplerian profile subtracted for simulation ME10t1T1h1 with $\Omega \tau=1$ (see Table 1 in Lobo Gomes et al. (submitted) for the details of the runs).

timescale, which is in agreement with previous studies (Fu et al. 2014; Les \& Lin 2015). We used thermal relaxation times ranging from $\Omega \tau=0.01$ to $\Omega \tau=10$ (see Fig. 3 ) and found the strongest secondary vortex for $\Omega \tau=0.1$. Les \& Lin (2015) explained that the nonmonotonic behavior is due to the fact that the vortex lifetime depends on (i) the decay timescale of the RWI, which decreases for increasing $\Omega \tau$, and (ii) the vortex growth time, which increases for values of up to $\Omega \tau=5.0$ and then decreases for larger values. Therefore the cooling properties of the disk play a crucial role for the generation and maintenance of these structures.

\section{Low Mass Planets}

We used global 2D-HD simulations, with the same cooling approach as in the previous section, to study the unsaturation of the horseshoe drag for type I migration (Lobo 


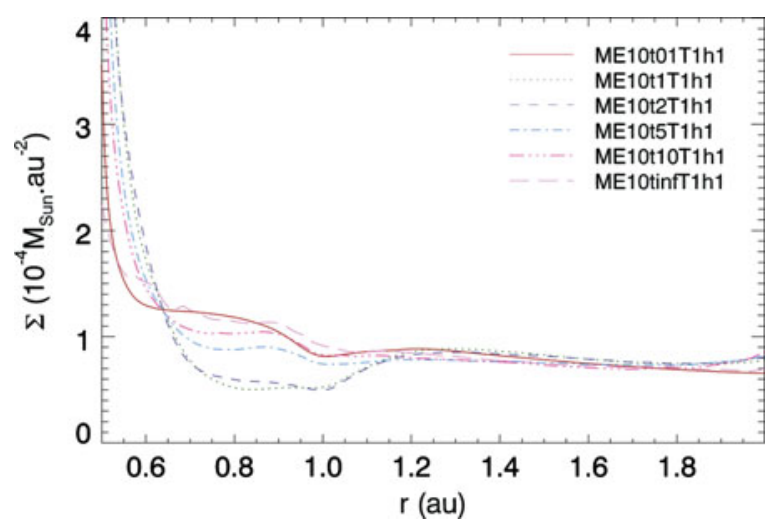

Figure 6. Surface density profiles averaged in azimuth at 2000 orbits and for a $10 M_{\oplus}$ planet, $\beta_{T}=1.0, h=0.1$, and the thermal relaxation timescales from solid brown to dashed pink (same as Fig.4): $\Omega \tau=[0.1,1.0,2,5,10, \infty]$.

Gomes et al., submitted). Radial stratification in combination with thermal relaxation can lead to the development of a CO, which generates and amplifies vortices (e.g., Klahr \& Hubbard 2014). Hydrodynamical instabilities can cause turbulence. The main goal of this study was to check whether turbulence-triggered viscosity due to the CO can sustain the unsaturation of the horseshoe drag, which is essential to balance or counteract the Lindblad torque. Figure 4 shows the torques as a function of the thermal relaxation timescale.

We found that the disk parameters that favour slow/outward migration oppose the amplification of vortices (see Lobo Gomes et al., submitted). Therefore this is not a good mechanism to prevent the saturation of the horseshoe drag. However, we observed that the planet perturbation leads to the formation of vortices in the horseshoe region. These vortices get amplified and excite spiral waves, leading to fast inward vortex migration. The continuous planet perturbation results in permanent vortex formation, see Figure 5 . The process of constant creation and amplification of vortices, that later migrate to the inner disk, depletes the matter in the inner side of the planet orbit, see Figure 6 . The planet remains trapped in the outer edge of this under-density. This result is a new promising mechanism to prevent the fast type I migration rates in regions of the disk that are convective overstable.

\section{Conclusions}

The CO was found to play a role for both high and low mass planet cases. For the former, the presence of the CO leads to the formation of weaker planet-induced vortices. The further evolution of the vortices, however, is not strongly dependent on the CO. The formation of a second generation of vortices was observed, nonetheless it is not linked to the presence of the CO. This second generation of vortices may explain the vortex in the Oph IRS48 system. We found also a strong dependence of the vortices lifetimes and birth times with the thermal relaxation timescale, in agreement with previous results. For the latter, the CO does not prevent the saturation of the horseshoe drag, in contrast, the disk parameters that favour CO seem to oppose the horseshoe drag unsaturation. Nevertheless, the formation of a planet trap was observed for the cases in which the CO was in action. The effect of continuous vortex formation and shedding inside of the planet appears to be a new interesting mechanism to trap planets. Detailed studies on how the 
efficiency depends of the susceptibility of the disk to CO (detailed slopes of T and Sigma) will have to follow including the possibilities that vortices do form spontaneously outside the planet orbit or even being triggered by additional planets.

\section{Acknowledgments}

H. Klahr and A. Lobo Gomes would like to thank financial support from the Deutsche Forschungsgemeinschaft (DFG), grant n. KL 1469/9-1. A. Lobo Gomes is also grateful for the support by the International Max Planck Research School at Heidelberg (IMPRS- HD). The simulations were performed on the THEO cluster at the Rechenzentrum Garching (RZG) of the Max Planck Society.

\section{References}

Andrews, S. M., Wilner, D. J., Hughes, A. M., Qi, C., \& Dullemond, C. P. 2010, ApJ, 723(2), 1241-1254

Asphaug \& Benz (1996)]Asphaug Alibert, Y., Mordasini, C., \& Benz, W. 2004, A\& A, 417, L25

Ataiee, S., Dullemond, C. P., Kley, W., Regály, Z., \& Meheut, H. 2014, A\&A, 572, A61

Balbus, S. A. \& Hawley, J. F. 1991, ApJ, 376, 214

Baruteau, C. \& Masset, F. 2008, ApJ, 672, 1054

Barge, P. \& Sommeria, J. 1995, A\&A, 295, L1

Casoli, J. \& Masset, F. S. 2009, ApJ, 703, 845

Fu, W., Li, H., Lubow, S., \& Li, S. 2014, ApJL, 788, L41

Ida, S. \& Lin, D. N. C. 2008, ApJ, 673, 487

Klahr, H. H. \& Bodenheimer, P. 2003, ApJ, 582, 869

Klahr, H. \& Bodenheimer, P. 2006, ApJ, 639, 432

Klahr, H. \& Hubbard, A. 2014, ApJ, 788, 21

Koller, J., Li, H., \& Lin, D. N. C. 2003, ApJL, 596, L91

Les, R. \& Lin, M.-K. 2015, MNRAS, 450, 1503

Lesur, G. \& Papaloizou, J. C. B. 2010, $A \& A$, 513, A60

Li, H., Lubow, S. H., Li, S., \& Lin, D. N. C. 2009, ApJL, 690, L52

Lobo Gomes, A., Klahr, H., Uribe, A. L., Pinilla, P., \& Surville, C. 2015, ApJ, 810, 94

Lobo Gomes, A., Klahr, H., Surville, C. \& Uribe, A. L. submitted, ApJ

Lovelace, R. V. E., Li, H., Colgate, S. A., \& Nelson, A. F. 1999, ApJ, 513, 805

Lyra, W. \& Mac Low, M.-M. 2012, ApJ, 756, 62

Lyra, W. \& Klahr, H. 2011, A\&A, 527, A138

Meheut, H., Yu, C., \& Lai, D. 2012, MNRAS, 422, 2399

Mordasini, C., Alibert, Y., \& Benz, W. 2009, A\& A, 501, 1139

Morohoshi, K. \& Tanaka, H. 2003, MNRAS, 346, 915

Ou, S., Ji, J., Liu, L., \& Peng, X. 2007, ApJ, 667, 1220

Paardekooper, S.-J. \& Mellema, G. 2006, A\&A, 459, L17

Paardekooper, S.-J. \& Papaloizou, J. C. B. 2009, MNRAS, 394, 2283

Petersen, M. R., Julien, K., \& Stewart, G. R. 2007a, ApJ, 658, 1236

Petersen, M. R., Stewart, G. R., \& Julien, K. 2007b, ApJ, 658, 1252

Raettig, N., Lyra, W., \& Klahr, H. 2013, ApJ, 765(2), 115

Regály, Z., Sándor, Z., Csomoś, P., \& Ataiee, S. 2013, MNRAS, 433, 2626

Tanaka, H., Takeuchi, T., \& Ward, W. R. 2002, ApJ, 565, 1257

Uribe, A. L., Klahr, H., Flock, M., \& Henning, T. 2011, ApJ, 736, 85

van der Marel, N., van Dishoeck, E. F., Bruderer, S., et al. . 2013, Science, 340, 1199

Ward, W. R. 1986, Icarus, 67, 164

-. 1988, Icarus, 73, 330 
-. 1997, Icarus, 126, 261

Whipple, F. L. 1972, in From Plasma to Planet, ed. A. Elvius (New York: Wiley Interscience Division), 211

Yu, C., Li, H., Li, S., Lubow, S. H., \& Lin, D. N. C. 2010, ApJ, 712, 198

Zhu, Z. \& Stone, J. M. 2014, ApJ, 795, 53 